PFC/ JA-85-37

\author{
Non-Thermal Electron Velocity Distribution Measured by \\ Electron Cyclotron Emission in Alcator C Tokamak \\ K. Kato and I. H. Hutchinson \\ Plasma Fusion Center \\ Massachusetts Institute of Technology \\ Cambridge, MA 02139 \\ October 1985
}

This work was supported by the U.S. Department of Energy Contract No. DE-AC02-78ET51013. Reproduction, translation, publication, use and disposal, in whole or in part by or for the United States government is permitted.

By acceptance of this article, the publisher and/or recipient acknowledges the U.S. Government's right to retain a non-exclusive, royalty-free license in and to any copyright covering this paper. 


\title{
Non-Thermal Electron Velocity Distribution Measured by Electron Cyclotron Emission in Alcator C Tokamak
}

\author{
K. Kato and I.H. Hutchinson \\ Plasma Fusion Center, Massachusetts Institute of Technology \\ Cambridge, Massachusetts, 02139, U.S.A.
}

\begin{abstract}
The non-thermal electron velocity distribution of lower hybrid RF heated plasmas in the Alcator $\mathrm{C}$ tokamak is deduced from measurements of electron cyclotron emission using a specialized vertical viewing optics arrangement. At a density of $\bar{n}_{e}=0.7 \times 10^{20} \mathrm{~m}^{-3}$, the reconstructed distribution function in the range $50 \mathrm{keV} \leq E \leq 230 \mathrm{keV}$ shows enhancement in the parallel direction with a perpendicular spread equivalent to $T_{\perp} \approx 30 \mathrm{keV}$. The line average energetic electron density in this energy range is approximately $7 \times 10^{15} \mathrm{~m}^{-3}$ during the RF.
\end{abstract}

PACS Numbers: 52.25.Ps, 52.55.Gb 
In this letter, we report on the non-thermal electron velocity distribution obtained from the vertical viewing $\mathrm{ECE}$ diagnostic on Alcator $\mathrm{C}\left(R=64 \mathrm{~cm}, a=16.5 \mathrm{~cm}, B_{T}=\right.$ $8 T$ ). In the past ECE measurements of the distribution were limited to a fitting of a few parameters(e.g. $T_{\perp}$ and $\left.T_{\|}\right)$; some of these measurements are reviewed in Ref.1. In comparison, by using our configuration, rather general distributions can be diagnosed in significant detail with a minimum of assumptions about their shape. Recent experiments using first harmonic absorption in vertical propagation have been performed on PLT ${ }^{2}$.

The theoretical principles of the present method are discussed in detail elsewhere ${ }^{3}$ but we summarize them here. When perpendicular electron cyclotron emission from a region of approximately constant magnetic field is observed, the frequency shift is entirely due to the relativistic mass increase factor, $\gamma: \omega=l \Omega / \gamma$, where $l$ is the cyclotron harmonic number and $\Omega$ is the fundamental rest-mass cyclotron frequency. In this specialized situation, it is possible to discriminate electrons according to their total energy, provided overlap of the neighboring harmonics does not occur.

The ECE intensity in this case yields information regarding the distribution of electrons in momentum space. Under the assumption of negligible absorption, the observed intensity is proportional to the emissivity, which can be written as an integral of $f(\vec{p})$ over the momentum pitch-angle, weighted by the single particle emissivity and corrected for finite plasma effects ${ }^{4}$. (The momentum is in natural units: $p=\gamma v / c$.) The unnormalized electron distribution function, $f(\vec{p})$, is expressed as

$$
f(\vec{p})=f_{p}(p) f_{\theta}(p, \theta)
$$


where $f_{p}(p)$ is the mean phase space density of electrons at $p$ and we choose

$$
f_{\theta}(p, \theta)=L \exp \left\{-\Lambda(p) \cos ^{2} \theta\right\}
$$

as the pitch-angle distribution, with a normalization $\int_{0}^{\pi} f_{\theta}(p, \theta) \sin \theta d \theta=2$. This choice of $f_{\theta}$ is a gaussian-like function along the circular resonance contour in momentum space, $p=$ const. The parameter to be fitted, $\Lambda$, determines the anisotropy of the distribution along the contour. A negative $\Lambda$ implies enhancement in the parallel direction(prolate distribution) while positive $\Lambda$ implies loss-cone type of anisotropy(oblate). This anisotropy factor, $\Lambda$, can be obtained independently of $f_{p}$ from ratios of two harmonic intensities of the same electron energy, for example, $I(3 \omega) / I(2 \omega)$. Then the absolute intensity of a single harmonic yields $f_{p}$. Perpendicular emission gives no information on any parallel asymmetry in the distribution. However, our symmetric $f(\vec{p})$ may be modified to model asymmetric distributions if necessary.

To isolate a region of constant magnetic field, the Alcator $\mathrm{C}$ tokamak plasma is viewed vertically from the top of the machine, through the center of the plasma. The spread of antenna pattern in the major radius direction is approximately $3 \mathrm{~cm}$ at the plasma center, which translates to a field variation of $5 \%$. In order to remove reflected radiation that could introduce spurious emission from other magnetic fields or propagation angles, a compact microwave viewing dump made of Pyrex ${ }^{5}$ was placed at the bottom of the vacuum chamber. This dump is essential because the spot size of the view is larger than the access port at the bottom. Tests using reproducible thermal ECE show the dump to be effective in removing approximately $90 \%$ of the unwanted radiation ${ }^{6}$.

The extraordinary mode frequency spectrum is obtained every $15 \mathrm{~ms}$ by a rapidscanning Michelson interferometer and liquid helium cooled InSb detector. The choice 
of extraordinary mode is motivated by its higher emissivity and narrower antenna pattern in our system(because of diffraction) compared to the ordinary mode. The entire optical train has been calibrated for relative frequency response in the range $100 \mathrm{GHz}$ to $900 \mathrm{GHz}$ using a mercury arc lamp. The absolute response is obtained from the second harmonic thermal emission of a plasma discharge of known electron temperature.

In Fig.1 we show the ECE spectrum obtained before and during lower hybrid RF heating at a density of $\bar{n}_{e}=0.7 \times 10^{20} \mathrm{~m}^{-3}$. Data were also taken on lower hybrid current drive shots. However, the excessive harmonic overlap due to the higher mean energy of the electron population makes the interpretation more difficult and a reliable distribution has not yet been obtained from them. Therefore, we do not discuss such plasmas here.

The thermal spectrum(broken line) shows that the dump is quite effective in suppressing the emission from outside the line of sight. The second harmonic emission $(440 \mathrm{GHz})$ peak has a width consistent with the antenna pattern. (Spectra of the second harmonic thermal emission without the dump show emission from regions all along a major diameter because of multiple reflections.)

The non-thermal spectrum(solid line) shows several notable features. The downshifted first harmonic emission is seen below $\Omega(220 G H z)$. The dip at $220 G H z$ is due to the bulk plasma thermal absorption. The narrow peak at $270 \mathrm{GH} z$ we believe to be because the right hand cut-off layer in the line of sight reflects the antenna pattern away from the dump and hence enhances the intensity observed. The dips at $380 \mathrm{GHz}$ and $560 \mathrm{GHz}$ are atmospheric water vapor absorption lines, which limit the energy range over which the spectrum can be interpreted quantitatively with full confidence. A dramatic discontinuity is seen just above $2 \Omega$. The substantially lower emission on the high frequency side is consistent with 
expectations of purely downshifted emission and attests to the effectiveness of the dump in suppressing reflected radiation. Overlapping third harmonic down-shifted radiation continues below the second harmonic rest mass frequency, $2 \Omega$, as indicated by the finite intensity just above it. This indicates that some unwrapping of the harmonics is required. Above $3 \Omega$, noise starts to dominate the features due to decreasing response of the detector in this frequency range.

The distribution function parameters are deduced, as described above, using a computer code which takes into account the mild harmonic overlap. The ratio $I(3 \omega) / I(2 \omega)$ is used to obtain $\Lambda$ and then $I(2 \omega)$ is used to obtain $f_{p}$. First harmonic is excluded from this analysis because of larger uncertainty in the calibration, decreased effectiveness of the dump at low $(<200 G \mathrm{~Hz})$ frequencies, and non-negligible plasma optical depth. The choice of $I(3 \omega) / I(2 \omega)$ limits the analysis in electron energy space to below $230 \mathrm{keV}$.

For the code analysis, the two water lines at $380 \mathrm{G} \mathrm{Hz}$ and $560 \mathrm{G} \mathrm{Hz}$ are filled in by linear interpolation between frequencies $364 \mathrm{GHz}$ and $398 \mathrm{GHz}$, and $519 \mathrm{GHz}$ and $601 \mathrm{GHz}$ (shown by dotted lines in Fig.1). The actual intensity is at most $15 \%$ higher than the interpolated value near $560 \mathrm{GHz}$ and within calibration uncertainty at $380 \mathrm{GHz}$. These estimates are based on observation of the general shape of the spectra obtained under different conditions such as different toroidal fields. The resonant electron energy range unaffected by interpolation is above $140 \mathrm{keV}$. In view of the previously mentioned right hand cut-off effect, the region of analysis is terminated before the right hand cut-off peak at $270 \mathrm{GHz}$.

The distribution function parameters, $f_{p}(p)$ and $\Lambda(p, \theta)$, obtained from the spectrum of Fig.1, are plotted versus resonant electron energy in Fig.2. The magnitude of $f_{p}$ is to be interpreted as the line average density $\left(\mathrm{m}^{-3}\right)$ over the $33 \mathrm{~cm}$ chord in the plasma. $f_{p}$ is 
decreasing but persistent all the way up to the $230 \mathrm{keV}$ limit. The anisotropy factor, $\Lambda$, is negative and in the range $-6 \leq \Lambda \leq-2$ above $50 \mathrm{keV}$. At $50 \mathrm{keV}$, a positive $\Lambda$ is obtained, probably due to uncertainties in the harmonic overlap estimate. Since the harmonic ratio is insensitive to $\Lambda$ for $\Lambda>0$, we artificially force $\Lambda=0$ for this energy. A relativistic maxwellian $(T \approx 80 \mathrm{keV})$ may be fitted to $f_{F}$ as shown by the straight line in Fig.2(a). This fit will be used to compute absorption coefficients discussed below.

To illustrate the distribution function, contours of constant $f(\vec{p})$ are plotted in $\left(p_{\|}, p_{\perp}\right)$ space in Fig.3. (The $50 \mathrm{keV}$ data are excluded.) This clearly shows the enhancement of the electron population in the parallel direction, as measured by other experiments ${ }^{7}$ and predicted by theory ${ }^{8}$. The bump at $p_{\|} \simeq 0.9$ is due to the bump in $f_{p}$, although, as the error bars indicate, it is probably not statistically significant.

The 'perpendicular temperature', $T_{\perp}$, as a function of $E_{\|}=m_{0} c^{2}\left\{\left(1+p_{\|}^{2}\right)^{1 / 2}-1\right\}$ is also obtainable from $f(\vec{p})$. Here it is defined such that the distribution along a $p_{\|}=$const. cross section best fits the expression,

$$
f\left(p_{\perp}, p_{\|}=\text {const. }\right)=A \exp \left\{-\frac{m_{\circ} c^{2}}{T_{\perp}\left(E_{\mid}\right)}\left(1+p_{\|_{i}}^{2}+p_{\perp}^{2}\right)^{1 / 2}\right\}
$$

The perpendicular temperature thus obtained is shown in Fig.4. The average value of $T_{\perp}$ is approximately $30 \mathrm{keV}$. Any trend in $T_{\perp}$ as a function of $E_{\mid}$is difficult to determine owing to the large scatter of the data which comes primarily from the bumpiness of $f_{p}$. The ECE radiation temperature, $T_{r}$, at the first harmonic, which is approximately equal to the 'perpendicular temperature' of the distribution if the optical depth is large, is also plotted in Fig.4, for the range $50 \mathrm{keV} \leq E \leq 500 \mathrm{keV}$. 
To compare $T_{\perp}$ and $T_{r}$, the absorption coefficient, $\alpha$, is calculated from the smoothed $f_{p}$ shown in Fig.2(a) and corresponding $\Lambda$ obtained from $I(2 \omega)$. The optical depth $\tau$ calculated in this way for $50 \mathrm{keV} \leq E \leq 230 \mathrm{keV}$ at the second harmonic is $\tau_{2} \leq 0.1$ so that the assumption of negligible absorption is justified. In the first harmonic, $\tau_{1} \approx 0.4$. Therefore, $T_{\perp}$ deduced from the first harmonic, $T_{\perp} \simeq T_{r} /\left(1-\exp \left(-\tau_{1}\right)\right)$, is consistent with that deduced from the second and the third harmonics, at least above $120 \mathrm{keV}$ (Fig.4). At lower energy, the proximity of the thermal resonance appears to affect the spectrum. The $30 \mathrm{keV}$ perpendicular temperature of the distribution seems reasonable compared with $T_{\perp} \approx 100 \mathrm{keV}$ estimated for current drive plasmas ${ }^{7}$, whose lower hybrid wave spectrum creates far more energetic electron tail and therefore higher $T_{\perp}$ than in the heating case ${ }^{8}$.

Finally, the energetic electron population for the range $50 \mathrm{keV} \leq E \leq 230 \mathrm{keV}$ is calculated to be $\bar{n}_{T} \approx 7 \times 10^{15} \mathrm{~m}^{-3}$. The fraction $\bar{n}_{T} / \bar{n}_{e} \approx 1 \times 10^{-4}$ is smaller than other estimates $^{9}$, but our estimate excludes energies below $50 \mathrm{keV}$ where a large fraction of the tail electrons are present.

In summary, we have successfully deduced an electron velocity distribution in the energy range $50-230 \mathrm{keV}$ using a much more detailed method of analysis than previously carried out on non-thermal ECE spectrum. The non-thermal electron distribution during lower hybrid heating discharge at $\bar{n}_{e}=0.7 \times 10^{20} \mathrm{~m}^{-3}$ in Alcator $\mathrm{C}$ is given as an example. Noteworthy features of the deduced distribution include enhancement in the parallel direction, effective temperature of the energetic distribution of approximately $80 \mathrm{keV}$, and $T_{\perp} \approx 30 \mathrm{keV}$. This $30 \mathrm{keV} T_{\perp}$ is in rough agreement with that deduced from the first harmonic intensity. The estimate of the energetic electron population density is $1 \times 10^{-4} \bar{n}_{e}$ in the range $50-230 \mathrm{keV}$. 
We thank all the other members of the Alcator group for their support, especially the RF section, Drs. Porkolab, Knowlton, Takase, and Texter. This work was supported by U.S. Department of Energy Contract No. DOE-AC02-78ET51013. 


\section{References}

${ }^{1}$ D.A. Boyd, Proc. 4th Int. Workshop in ECE and ECRH, Rome, March 28-30, 1984, p.145.

${ }^{2}$ E. Mazzucato, P. Efthimion, and I. Fidone, Princeton University Report, PPPL-2122 (1984), to be published in Nucl. Fusion.

${ }^{3}$ I.H. Hutchinson and K. Kato, M.I.T. Report PFC/JA-85-15 (1985). submitted to Nucl. Fusion.

${ }^{4}$ H.P. Freund and C.S. Wu, Phys. Fluids, 20 (1977) 963.

${ }^{5}$ K. Kato and I.H. Hutchinson, M.I.T. Report PFC/RR-84-11 (1984).

${ }^{6}$ K. Kato and I.H. Hutchinson, IEEE Int'l Conf. on Plasma Science, Cat. No. 85 CH2199 - 8, Pittsburgh, PA, June 3-5, 1985.

${ }^{7}$ S. von Goeler, et. al., Princeton University Report, PPPL-2225(1985), submitted to Nucl. Fusion. S. Texter, et. al., Bull. Am. Phys. Soc. Vol. 39, No.9(1985)p.1494.

${ }^{8}$ C.F.F. Karney and N.J. Fisch, Phys. Fluids, 22(1979)1817.

${ }^{9}$ M. Porkolab, et. al., Phys. Rev. Lett. 53(1984) 1229. 


\section{Figure Captions}

1. Extraordinary mode ECE spectra for non-thermal emission during lower hybrid RF heating $\left(\bar{n}_{e}=0.7 \times 10^{20} m^{-3}, I_{p}=180 k A, P_{R F}=400 k W, t=260 m s\right)$ shown by the solid line, and thermal emission just prior to RF injection of the same discharge $\left(\bar{n}_{e}=1.2 \times\right.$ $10^{20} \mathrm{~m}^{-3}, I_{p}=270 \mathrm{k} A, t=180 \mathrm{~ms}, T_{e o}=2.5 \mathrm{keV}$.) shown by the broken line. Deuterium discharge, $B_{T}=8 T, f_{c e}=220 G \mathrm{~Hz}$, frequency resolution $\simeq 15 G \mathrm{~Hz}$.

2. Distribution function parameters, (a) $f_{p}$ and (b) $\Lambda$ plotted as a function of the total electron energy. The range of energy for which interpolated data was used is indicated in (b).

3. Distribution function contour plot for $0.45 \leq p \leq 1.05$ (corresponding to $50 k e V \leq E \leq$ $230 \mathrm{keV})$ contour magnitudes are given in $\log (f(\vec{p}))$.

4. Perpendicular temperature, $T_{\perp}$, obtained from the distribution function(points), and radiation temperature, $T_{r}$, obtained from the first harmonic intensity(solid line) plotted versus the resonant electron energy. Broken line indicates the estimated perpendicular temperature from $T_{r}$ and $\tau_{1}$. 


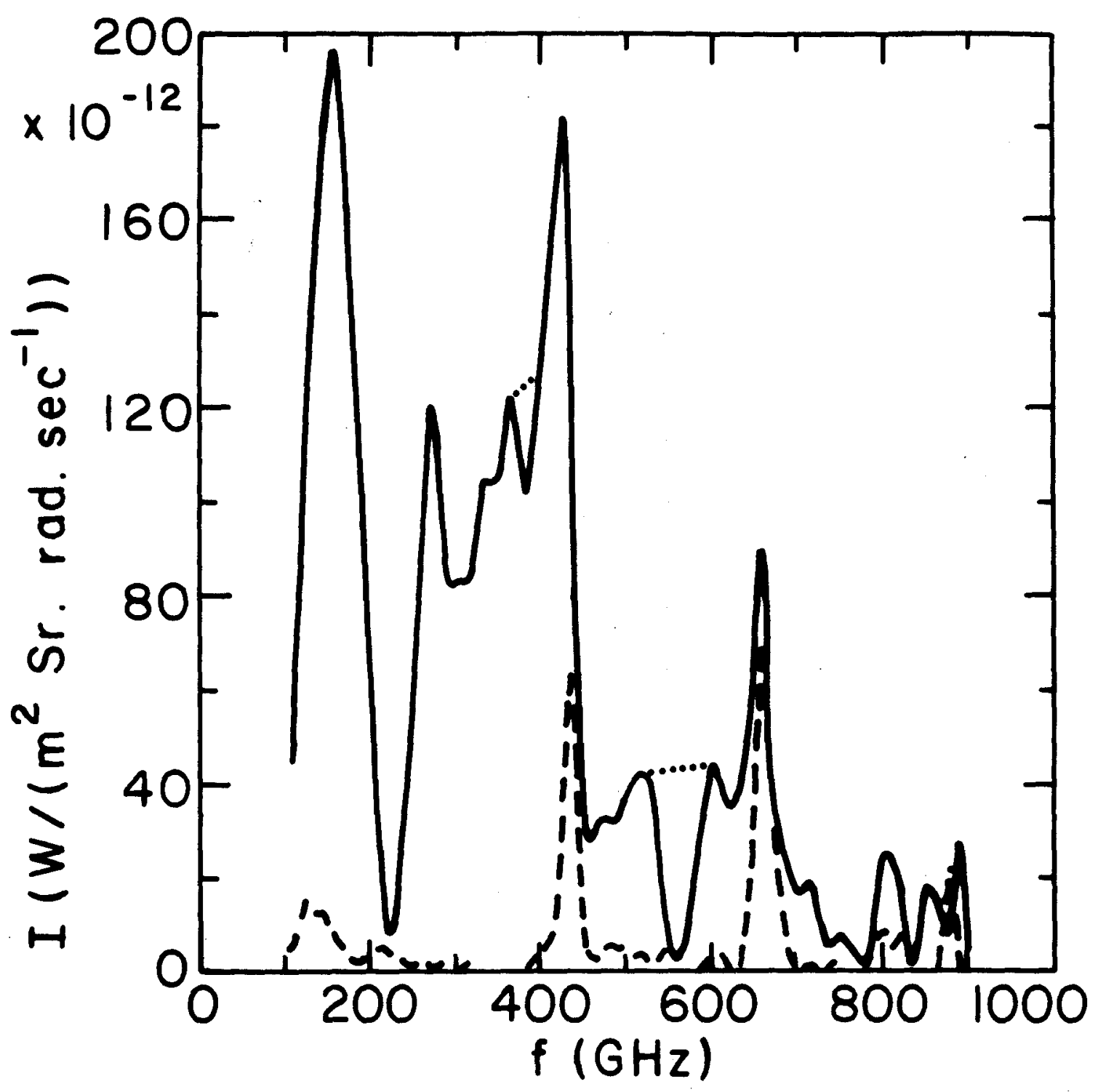

Figure 1 


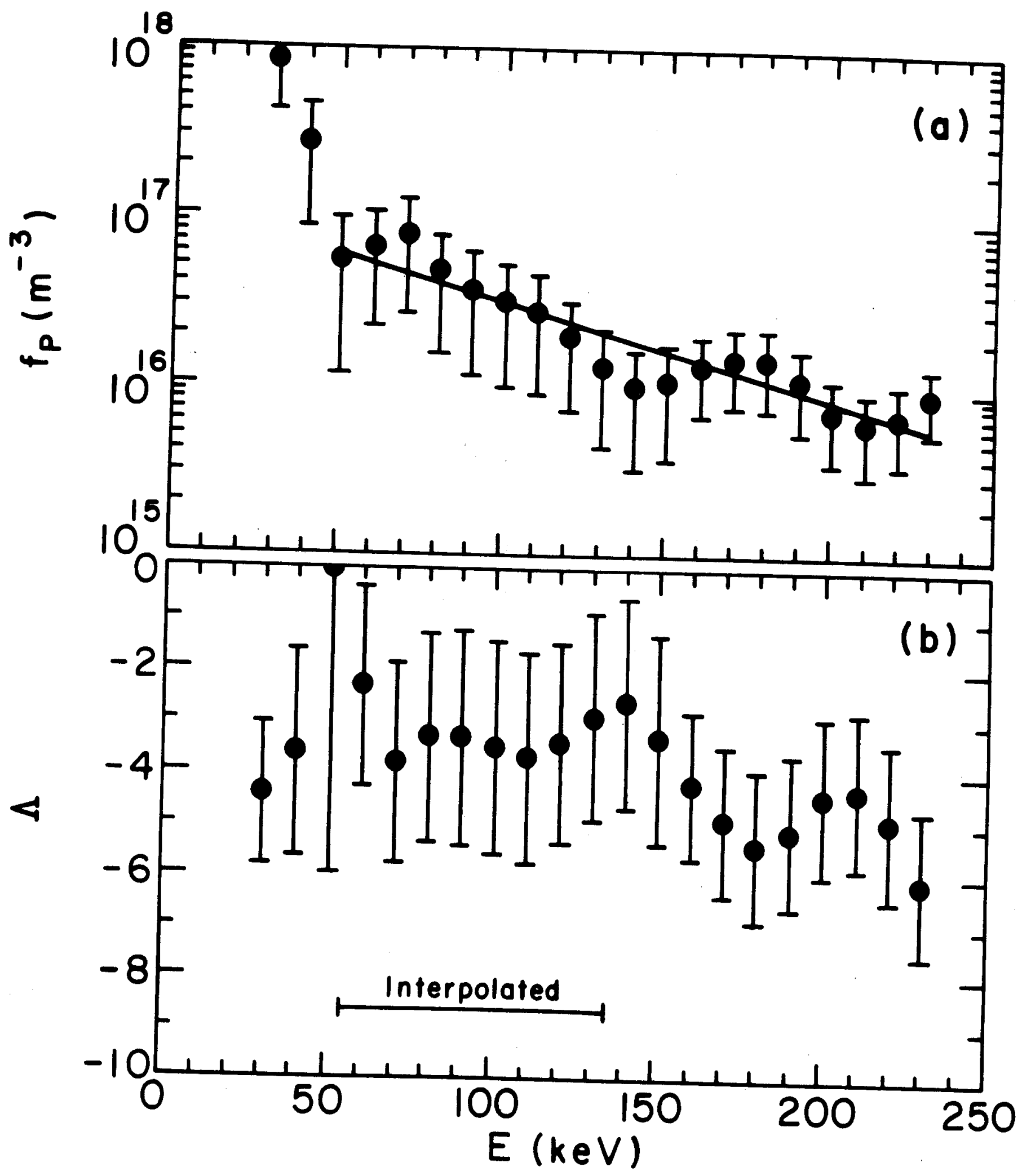

Figure 2 


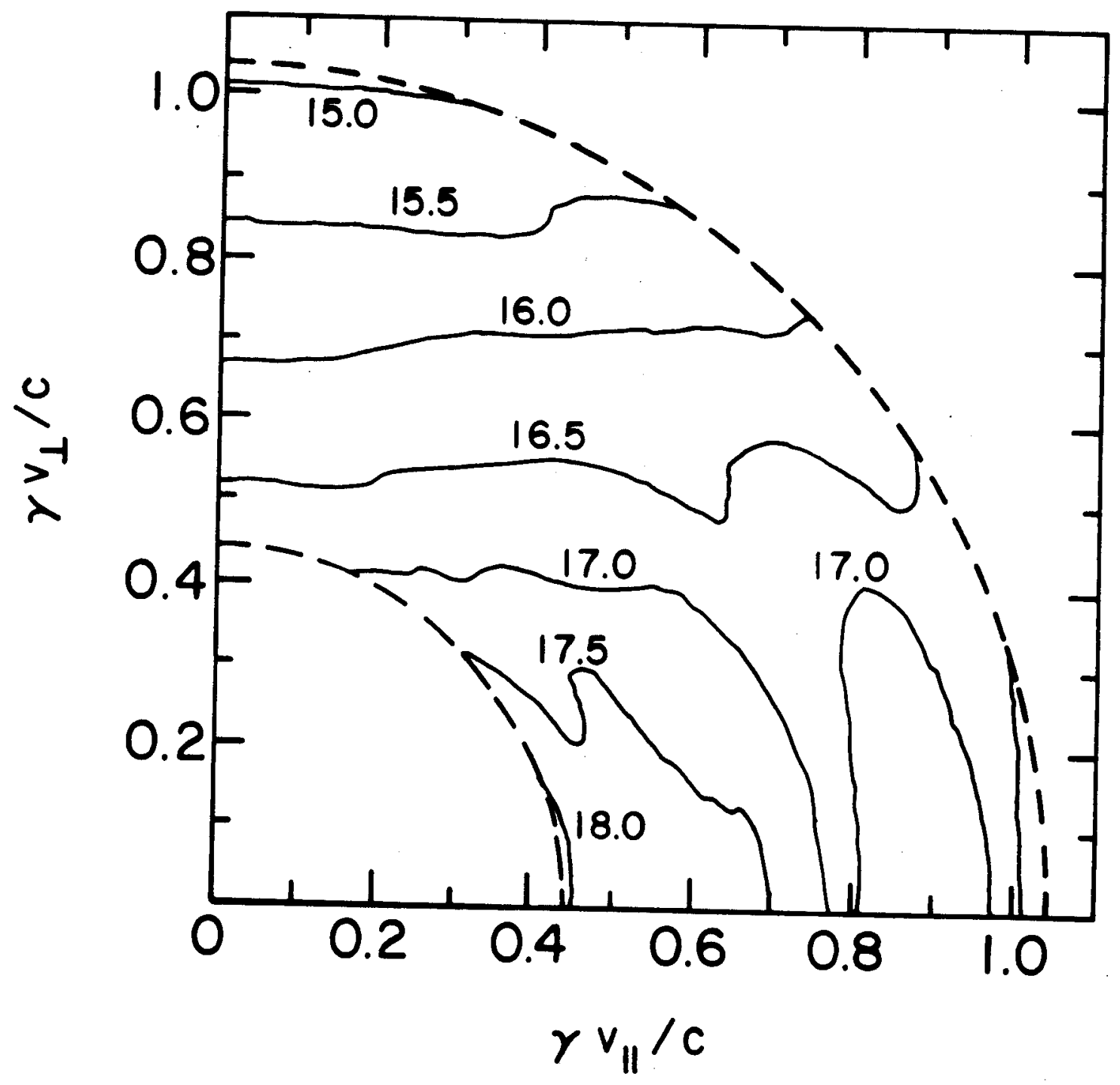

Figure 3 


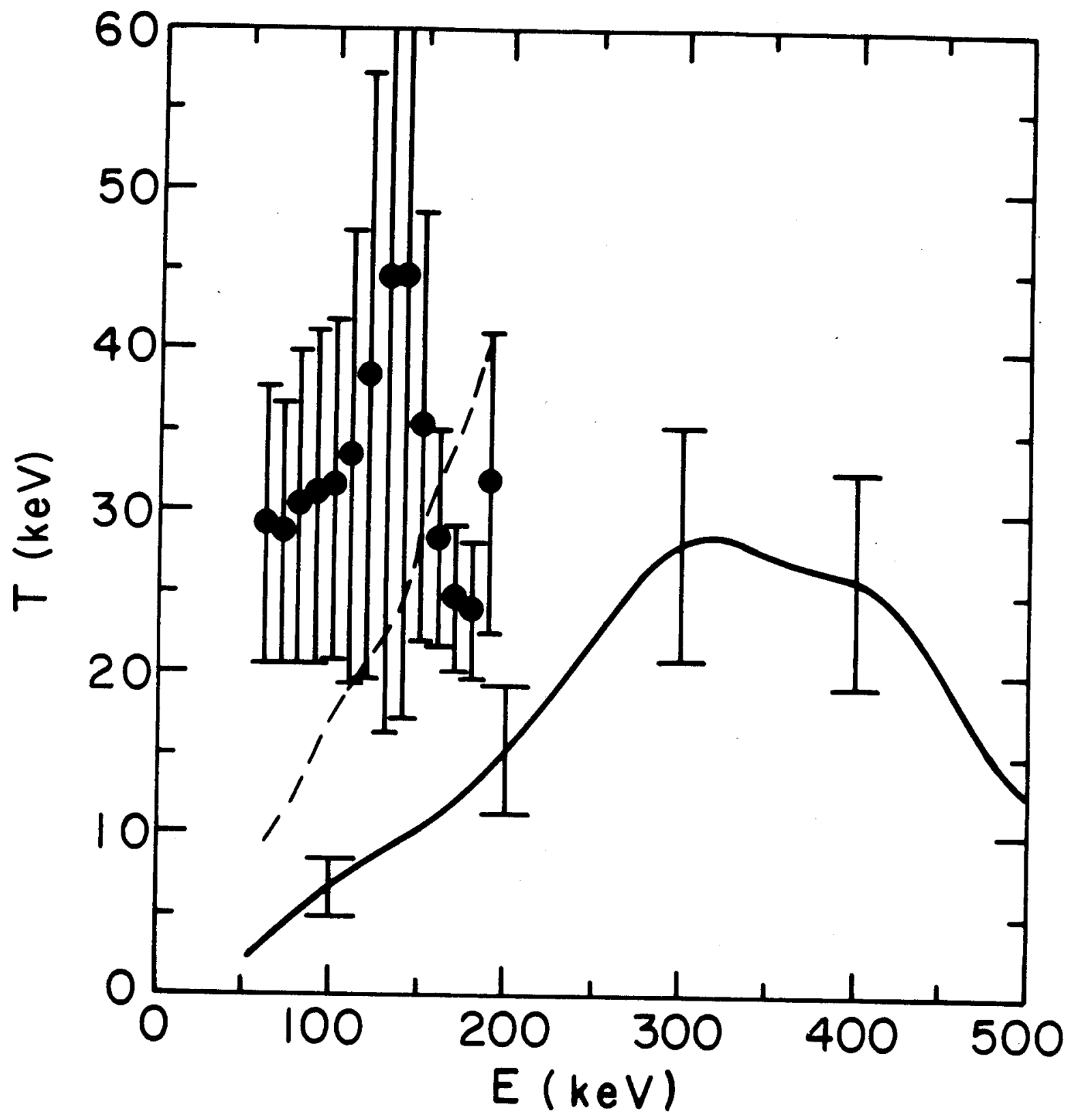

Figure 4 